\title{
From the inside out: An epibiotic Bdellovibrio predator with an
} expanded genomic complement

Christoph M. Deeg ${ }^{1}$, Tan T. Le ${ }^{1}$, Matthias M. Zimmer ${ }^{2 \$}$, \& Curtis A. Suttle*1,2,3,4

Author affiliation: University of British Columbia, Department of 1: Microbiology and Immunology, 2: Earth, Oceans and Atmospheric

Sciences, 3: Botany; 4: Institute for the Oceans and Fisheries

*:Corresponding author: suttle@science.ubc.ca;

\$: M.Z. currently at Helmholtz-Institute for RNA-based Infection Research, Würzburg, Germany

\section{Abstract}

Bdellovibrio and like organisms are abundant environmental predators of prokaryotes that

12 show a diversity of predation strategies, ranging from intra-periplasmic to epibiotic predation.

13 The novel epibiotic predator Bdellovibrio qaytius was isolated from a eutrophic freshwater pond

14 in British Columbia, where it was a continual part of the microbial community. Bdellovibrio

15 qaytius was found to preferentially prey on the beta-proteobacterium Paraburkholderia

16 fungorum. Despite its epibiotic replication strategy, B. qaytius encodes a complex genomic

17 complement more similar to periplasmic predators as well as several biosynthesis pathways not

18 previously found in epibiotic predators. Bdellovibrio qaytius is representative of a widely

19 distributed basal cluster within the genus Bdellovibrio, suggesting that epibiotic predation might

20 be a common predation type in nature and ancestral to the genus.

\section{Introduction}

Biotic factors regulating bacterial populations in nature are often assumed to be viral lysis and zooplankton grazing (1); however, an underappreciated cause of mortality is predation by other prokaryotes. Such predators, collectively referred to as Bdellovibrio and like organisms (BALOs), have evolved several times independently and deploy a variety of "hunting strategies".

27 Many facultative predators with broad host ranges, such as Ensifer adhaerens and Myxococcus xanthus, deploy a "wolfpack strategy" where a prey cell is surrounded by several predators and lysed $(2,3)$. Other, more specialized obligate predators have a narrower host range and specific

30 predation strategies; for example, Bdellovibrio spp. enters the periplasm of the prey cell to 31 consume the prey's cytoplasm $(4,5)$. 
Bdellovibrio spp. are delta-proteobacteria predators that use a biphasic lifestyle comprising an attack phase, in which a small, highly motile flagellated cell seeks out prey, and a growth phase, characterized by the predator penetrating the outer membrane of the prey cell and consuming its cytoplasm (5). During the growth phase, the predator forms a characteristic structure in the prey's periplasm known as the bdelloplast, which consists of a rounded, osmotically stabile outer membrane of the prey cell and several replicating Bdellovibrio cells. The bdelloplast continues to grow until the resources of the prey cell are exhausted and culminates in the septation and release of several to dozens of new attack-phase cells. This dichotic lifestyle switch is mediated by a highly expressed riboswitch in B. bacterivorous (6). The related genera Bacteriovorax and Predibacter are in the family Bacteriovoracea, which is a sister family to the prototypical Bdellovibrionacea within the order Bdellovibrionales (7).

Curiously, the alpha-proteobacteria genus Micavibrio, which is unrelated to the Bdellovibrionales leads a remarkably similar lifestyle to Bdellovibrio species, with high prey specificity. However, these bacteria prey in an epibiotic fashion on the outside of the prey cell instead of penetrating into the periplasm (8). Due to their similar lifestyles, Micavibrio spp. are included into the BALOs.

Recently, an isolate of a newly described species, Bdellovibrio exovorus, in the family Bdellovibrionacea that is closely related to periplasmic bdelloplast-forming Bdellovibrio species, was shown to have an extremely narrow host range, and employ a different epibiotic replication strategy (9). In the attack-phase, cells of Bdellovibrio exovorus resemble those of other Bdellovibrio isolates; whereas, in growth-phase the cells do not penetrate into the cytoplasm, but stay attached to the outside of the prey, strongly resembling Micavibrio species. Further, in growth-phase, B. exovorus does not induce a bdelloplast and seems to extract the cytoplasmic contents of the prey across both membranes. Once the resources of the prey are exhausted, growth-phase result in binary fission releasing two progeny attack-phase cells. The comparatively small genome of $B$. exovorus has been linked to its epibiotic predation strategy and reductionist evolution from an ancestor capable of intra-periplasmic replication (9-11). Bdellovibrio qaytius is the second epibiotic predator within the genus Bdellovibrio. Its genomic complement, phylogenetic placement and environmental distribution broaden our understanding of the ecology and evolution of this genus. 


\section{Materials and Methods}

An isolate of a lytic bacterium, here named Bdellovibrio qaytius sp. nov., was obtained

from a water sample collected near the sediment surface of a eutrophic pond in Nitobe Memorial Garden at the University of British Columbia, Canada $\left(49^{\circ} 15^{\prime} 58^{\prime \prime} \mathrm{N}, 123^{\circ} 15^{\prime} 34^{\prime \prime} \mathrm{W}\right)$. As part of a bioassay for pathogens infecting heterotrophic protists a subsample of the water was inoculated into modified DY-V artificial freshwater medium with yeast extract and a wheat grain (12).

\section{Genome sequencing}

For PacBio sequencing, exponentially growing mixed cultures containing B. qaytius as well as Bodo saltans NG1 and its virus BsV were centrifuged in a Sorvall SLC-6000 for 20 min and $5000 \mathrm{rpm}$ at $4^{\circ} \mathrm{C}$ to remove eukaryotic cells (13). Particles in the supernatant concentrated approximately 100-fold by tangential flow ultrafiltration with at 30kDa cut-off (Vivaflow 200, PES) cartridge. To concentrate the cells further they were centrifuged at $28,000 \mathrm{rpm}, 15^{\circ} \mathrm{C}$ for 8 $\mathrm{h}$ in a Beckman ultracentrifuge using a Ti90 fixed-angle rotor (Beckman-Coulter, Brea, California, USA), and then sedimented onto a $40 \%$ Optiprep $50 \mathrm{mM}$ Tris-Cl, $\mathrm{pH} 8.0,2 \mathrm{mM}$

$78 \mathrm{MgCl}_{2}$ cushion for $30 \mathrm{~min}$ at $28,000 \mathrm{rpm}$, and $15^{\circ} \mathrm{C}$ in a SW40Ti swing-out rotor. The Optiprep gradient was created by underlaying a 10\% Optiprep solution in $50 \mathrm{mM}$ Tris-Cl, pH 8.0, $2 \mathrm{mM}$ $\mathrm{MgCl}_{2}$ with a $30 \%$ solution followed by a $50 \%$ solution and equilibration overnight at $4{ }^{\circ} \mathrm{C}$. One $\mathrm{ml}$ of concentrate from the $40 \%$ cushion was added atop the gradient and the concentrate was

82 fractionated by centrifugation in an SW40 rotor for $4 \mathrm{~h}$ at $25000 \mathrm{rpm}$ and $18^{\circ} \mathrm{C}$. The fraction

83 corresponding to the pathogen was extracted from the gradient with a syringe and washed twice

84 with $50 \mathrm{mM}$ Tris-Cl, $\mathrm{pH} 8.0,2 \mathrm{mM} \mathrm{MgCl}_{2}$ followed by centrifugation in an SW40 rotor for 20

$85 \mathrm{~min}$ at $7200 \mathrm{rpm}$ and $18^{\circ} \mathrm{C}$ and were finally collected by centrifugation in an SW40 rotor for 30 $86 \mathrm{~min}$ at $7800 \mathrm{rpm}$ and $18^{\circ} \mathrm{C}$. Purity of the concentrate was verified by fluorescence vs SSC of

87 SYBR-Green stained samples (Invitrogen Carlsbad, California, USA) on a FACScalibur flow 88 cytometer (Becton-Dickinson, Franklin Lakes, New Jersey, USA). High molecular weight 89 genomic DNA was extracted using phenol-chloroform-chloroform extraction. Length and purity 90 were confirmed by gel electrophoresis and by using a Bioanalyzer 2100 with the HS DNA kit 91 (Agilent Technology). PacBio RSII 20kb sequencing was performed by the sequencing center of 92 the University of Delaware. Reads were assembled using PacBio HGAP3 software with $20 \mathrm{~kb}$ 
seed reads resulting in a single contig of 3,376,027 bp, $97.08 \times$ coverage, $99.92 \%$ called bases and a consensus concordance of $99.9954 \%$ (14).

\section{Propagation and host range studies}

Plaque assays were performed by mixing $0.5 \mathrm{ml}$ putative host cultures in logarithmic growth stage and $10 \mu \mathrm{l}$ of Bdellovibrio qaytius stock culture with $4.5 \mathrm{ml}$ molten $0.5 \%$ DY-V agar and incubation for 48h. Propagation of Bdellovibrio qaytius in liquid culture was monitored by PCR with custom primers set specific to B. qaytius 16S rDNA (Forward-5'AGTCGAACGGGTAGCAATAC-3'， Reverse-5'-CTGACTTAGAAGCCCACCTAC-3') as well as a BALO-specific primer set by Davidov et al. (7). To obtain a pure isolate of prey cells present in the mixed microbial assemblage, culture samples were streaked onto a DY-V agar plate and incubated at room temperature. Distinct colonies were picked and propagated in liquid DY-V medium. Propagation of Bdellovibrio qaytius using these cultures as hosts was confirmed by PCR. The identity of the prey cell cultures was confirmed by universal 16S rDNA Sanger sequencing CCGYCAATTYMTTTRAGTTT-3') (15). To clean up the predator culture, E. coli (Thermo Fisher) cells were grown in LB medium and pelleted at 3,900 x g (4500 rpm) for $10 \mathrm{~min}$, washed with $10 \mathrm{ml}$ of HEPES/CaCl2 buffer ( $25 \mathrm{mM}$ HEPES, $2 \mathrm{mM} \mathrm{CaCl} 2$ ), centrifuged in a fixed angle rotor centrifuge at 3,900 x g for $5 \mathrm{~min}$, and re-suspended in $19 \mathrm{ml}$ of $\mathrm{HEPES} / \mathrm{CaCl} 2 \mathrm{buffer}$. This cell suspension was then inoculated with $1 \mathrm{ml}$ of $0.8-\mu \mathrm{m}$ PVDF membrane filtered lysate of the Bdellovibrio containing culture and B. qaytius propagation was monitored by PCR. Bdellovibrio remained viable at $4^{\circ} \mathrm{C}$ storage for up to two years and glycerol stocks of the native community containing Bdellovibrio as well as an inoculated E.coli TOP10 culture was stored at $-80^{\circ} \mathrm{C}$ for archival purposes.

\section{Environmental sampling}

The presence of B. qaytius in the Nitobe-Garden pond was determined in 20-L water samples that were taken bimonthly during spring and summer 2017 filtered through GF-A filters (Millipore, Bedford, MA, USA; nominal pore size $1.1 \mu \mathrm{m}$ ) laid over a $0.8-\mu \mathrm{m}$ pore-size PES membrane (Sterlitech, Kent, WA, USA). The remaining particulate material was concentrated into $250 \mathrm{ml}$ using a 30-kDa MW cut-off tangential flow filtration cartridge (Millipore, Bedford, MA, USA). DNA from these concentrates was extracted using phenol-chlorophorm extraction 
123 and subjected to PCR using Bdellovibrio qaytius specific 16S rDNA primers to confirm its 124 presence.

Microscopy

\section{Negative staining transmission electron microscopy}

Cultures of Escherichia coli TOP10 were inoculated with B. qaytius at two hour time intervals and infected cultures, as well as an uninfected control were diluted tenfold and fixed in $4 \%$ glutaraldehyde. Next, the samples were applied to the carbon side of formvar carbon-coated 400-mesh copper grids (TedPella, CA, USA) and incubated at $4^{\circ} \mathrm{C}$ in the dark overnight under $13230 \mathrm{~s}$.

\section{Ultra-thin sectioning transmission electron microscopy}

For higher resolution images, cells of E. coli infected with Bdellovibrio qaytius were harvested at at $4 \mathrm{~h}$ intervals, as well as from uninfected control cultures. Cells from $10 \mathrm{ml}$ of culture were pelleted at $5000 \mathrm{xg}$ in a Beckmann tabletop centrifuge using a fixed angle rotor. The

137 pellet was resuspended in $0.2 \mathrm{M}$ Na-cacodylate buffer, $0.2 \mathrm{M}$ sucrose, 5\% EM-grade 138 glutaraldehyde, $\mathrm{pH} 7.4$ and incubated for $2 \mathrm{~h}$ on ice. After washing in $0.2 \mathrm{M} \mathrm{Na-cacodylate}$ buffer, cells were post-fixed with $1 \%$ Osmium tetroxide. Samples were dehydrated through water/ethanol gradients and ethanol was substituted by acetone. Samples were embedded in an equal part mixture of Spurr's and Gembed embedding and the resin was polymerized at $60^{\circ} \mathrm{C}$ overnight. Fifty-nm thin sections were prepared using a Diatome ultra $45^{\circ}$ knife (Diatome,

143 Switzerland) on an ultra-microtome. The sections were collected on a 400x copper grid and 144 stained for $10 \mathrm{~min}$ in $2 \%$ aqueous uranyl acetate and 5 min in Reynold's lead citrate. Image data 145 were recorded on a Hitachi $\mathrm{H} 7600$ transmission electron microscope at $80 \mathrm{kV}$. Image $\mathrm{J}$ 146 (RRID:SCR_003070) was used to compile all TEM images. Adjustments to contrast and 147 brightness levels were applied equally to all parts of the image.

\section{Fluorescence In Situ Hybridization Epifluorescence Microscopy}

To confirm epibiotic predation, cultures for fluorescence in-situ hybridization (FISH)

150 were prepared as outlined below. Two 10-ml volumes of E. coli TOP10 were centrifuged at 3900

$151 \mathrm{xg}$ in a Beckman tabletop fixed angle centrifuge (4500 rpm) for $10 \mathrm{~min}$, washed with $5 \mathrm{ml}$ of $152 \mathrm{HEPES} / \mathrm{CaCl} 2 \mathrm{buffer}$, centrifuged at $3900 \mathrm{~g}$ for $5 \mathrm{~min}$, and re-suspended in $9 \mathrm{ml}$ of $153 \mathrm{HEPES} / \mathrm{CaCl} 2$ buffer. One $\mathrm{ml}$ of $B$. qaytius containing culture was added to the resuspended $E$. 
154 coli while another served as a control. Both cultures were incubated at room temperature for $24 \mathrm{~h}$ 155 and were centrifuged again at $3900 \mathrm{x} \mathrm{g}(4500 \mathrm{rpm})$ for $10 \mathrm{~min}$, washed with $10 \mathrm{ml}$ of PBS, 156 centrifuged at 3,900 x g for $5 \mathrm{~min}$, and re-suspended in $5 \mathrm{ml}$ of PBS. Two $\mathrm{ml}$ of the cultures were

157 fixed in a 1:3 dilution of $10 \%$ buffered formalin $(\mathrm{pH} 7.0 ; 10 \mathrm{ml}$ of 37\% formaldehyde, $0.65 \mathrm{~g}$ $158 \mathrm{Na} \square \mathrm{HPO} \square, 0.4 \mathrm{~g} \mathrm{NaH}_{2} \mathrm{PO}_{4} .90 \mathrm{ml}$ of Milli-QTM $\mathrm{H} 2 \mathrm{O}$ ) at $4^{\circ} \mathrm{C}$ for $3 \mathrm{~h}$. Cells were then 159 centrifuged again at 3,900 x g, washed twice in $10 \mathrm{ml}$ of PBS, re-suspended in $10 \mathrm{ml}$ of a 160 mixture of PBS and 96\% EtOH (1:1), and vortexed. In order to localize the predator an Alexa161488 tagged probe specific to Bdellovibrio $16 \mathrm{~S}$ rDNA was designed (5'162 /5Alexa488N/TGCTGCCTCCCGTAGGAGT-3') based on Mahmoud et al. which also served 163 as a template for the incubation protocol (16). Ten $\mu \mathrm{l}$ of sample was spotted onto a $70 \%$ EtOH164 cleaned slide, dried at room temperature, and then taken through a dehydration series of 50\%, $16580 \%$, and 95\% EtOH. $25 \mu \mathrm{l}$ of the hybridization master mix (20 mM Tris- $\mathrm{HCl}$ [pH 7.4], $0.1 \%$ $166 \mathrm{SDS}, 5 \mathrm{mM}$ EDTA, $0.8 \mathrm{M} \mathrm{NaCl}, 37 \%$ formalin, $1 \mathrm{ng} / \mu \mathrm{l}$ of final probe concentration) was added 167 onto the sample. A cover glass was placed onto each sample and the slides incubated for $2 \mathrm{~h}$ at $16846^{\circ} \mathrm{C}$. With the cover slip removed, the slides were subsequently submerged into a bath of wash 169 buffer and incubated at $48^{\circ} \mathrm{C}$ for $30 \mathrm{~min}$. Slides were rinsed with sterile deionized $\mathrm{H} 2 \mathrm{O}$ and dried 170 at room temperature. A drop of ProLongTM Diamond Antifade Mountant with DAPI (4,6171 diamidine-2-phenylindole) was spotted onto a new cover glass and placed on the sample. Finally, 172 the slides were incubated at room temperature in the dark for $24 \mathrm{~h}$ prior to observation on an 173 Olympus FV 1000 system.

\section{Annotation}

176 using the automated NCBI Prokaryotic Genome Annotation Pipeline (PGAAP). In parallel, open 177 reading frames were predicted using GLIMMER (RRID:SCR_011931) with default settings 178 (17). Translated proteins were analyzed using BLASTp, CDD RPS-BLAST and pfam HMMER. 179 These results were used to refine the PGAAP annotation. Signal peptides and trans-membrane 180 domains were predicted using Phobius (18). The annotated genome is available under the 181 accession number CP025544. Metabolic pathways were predicted using the Kyoto Encyclopedia 182 of Genes and Genomes (KEGG RRID:SCR_012773) automatic annotation server KAAS and 183 Pathway Tools (RRID:SCR_013786) (19, 20). 


\section{Phylogenetic analysis}

Full length 16S rDNA sequences of completely sequenced isoaltes of Bdellovibrio spp., as well as full-length uncultured top BLAST hits were downloaded from NCBI. Alignments of rDNA sequences were performed in Geneious R9 (RRID:SCR_010519) using MUSCLE with default parameters (RRID:SCR_011812)(21). Maximum likelihood trees were constructed with RAxML ML search with 1000 rapid bootstraps using GTR+GAMMA (22).

190 Phylogenetic analysis of the genome content by orthologous gene clusters was performed by 191 OrthoMCL (RRID:SCR_007839) (23) using whole genome sequences downloaded from NCBI. 192 OrthoMCL was run with standard parameters (Blast E-value cutoff $=10-5$ and mcl inflation 193 factor $=1.5$ ) on all protein-coding genes of length $\geq 100$ aa. This resulted in the definition of 4242 distinct gene clusters.

\section{Results}

\section{Isolation, host range and distribution}

A lytic pathogen of bacteria was collected in a mixed microbial assemblage from a temperate eutrophic pond in southwestern British Columbia, Canada. Based on full-genome sequencing and electron microscopy of the infection cycle the pathogen was determined to be a new species of bacterium, here named Candidatus Bdellovibrio qaytius (subsequently referred to as B. qaytius), after "q $\square \mathrm{a}$ :yt" ("kill it") in hən $\square \mathrm{q} \square$ əmin $\square$ əm $\square$ the language of the Musqueam tribe of Coast Salish, the indigenous peoples from who's territory it was isolated. B. qaytius propagated in a mixed microbial assemblage from the sample site (Supplementary Figure 1). Additionally, $B$. qaytius could also be propagated on a specific isolates from this assemblage that was identified as the beta-proteobacterium Paraburkholderia fungorum where high numbers of putative attack-phase B. qaytius cells could be observed under phase-contrast microscopy (Supplementary Figure 1). Inoculation of B. qaytius in cultures of Pseudomonas fluorescence, 210 PCR signals after propagation, presumable due to carry-over(Supplementary Figure 1). 211 Similarly, small clear plaques were only observed on plates of $P$. fungorum, but not on plates of

212 Pseudomonas fluorescence, or E. coli (Figure 1, Supplementary Figure 2). Bdellovibrio qaytius 213 was detected at several time points in DNA extracted from water concentrates from Nitobe 
214 Gardens UBC in 2017, four years after the initial isolation, indicating the population persists in 215 the pond (Supplementary Figure 3).



217 Figure 1 Plaque assay. Bdellovibrio qaytius added to plates of Paraburkholderia fungorum on the left, untreated control on the right. Arrow indicate location of plaques $24 \mathrm{hpi}$.

221 that are about $1 \mu \mathrm{m}$ by $0.4 \mu \mathrm{m}$ in size and exhibit a sheathed flagellum (Figure $2 \mathrm{~A}$ ). Once attack-

222 phase cells contact a prey cell, they attach irreversibly and form a broad predatory synapse and

223 discard the flagellum (Figure 2 B). No invasion of the prey cell was observed during growth-

224 phase, nor were bdelloplasts, implying that Bdellovibrio qaytius is an epibiotic predator (Figure

225 2). The growth-phase cell attached to the prey cell empties the cytoplasm of the host cell, leaving 226 behind an empty ghost cell (Figure 2 C,E,G). Simultaneously, the growth-phase Bdellovibrio cell 227 grows in size and once the resources of the prey cell are exhausted, the growth-phase cumulates 228 in binary fission and the production of two offspring attack-phase cells that repeat actively 229 searching for new prey cells by rapid locomotion. Throughout the growth-phase, the cell 230 membranes of the prey as well as the predator remain intact and instead of periplasmic invasion, 231 an electron dense layer is observed on both the prey's and predator's membranes suggesting that 232 a high concentration of effector molecules like transmembrane transporters are likely recruited to 233 these sited to facilitate predation (Figure 2 F). Predation was dependent on the growth-phase of 234 the host cell with cells in logarithmic growth supporting the highest B. qaytius concentrations. 

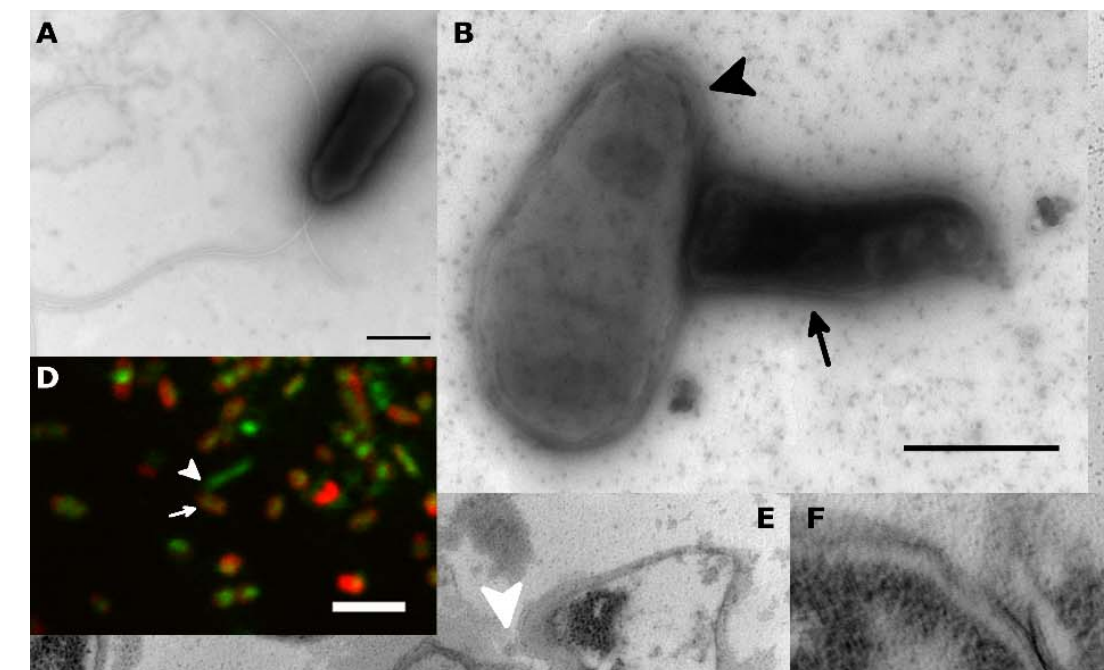

c.
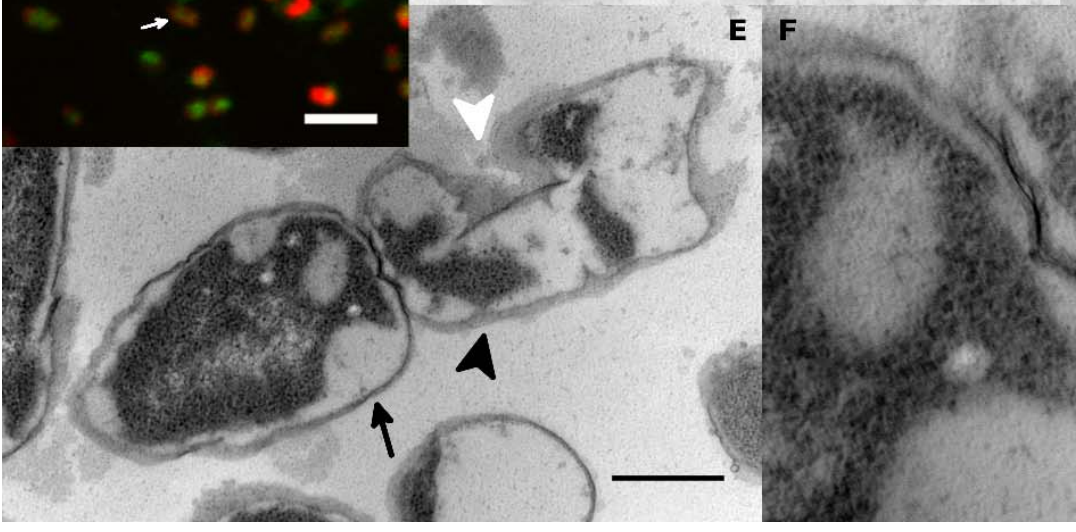

Figure 2 Bdellovibrio qaytius predation strategy and replication. A: Negative staining electron micrograph of an attack-phase cell showing the characteristic sheathed flagellum. B: Negative staining electron micrograph of an early growth-phase cell (arrow) attaching to a prey cell (arrow head) with a broad predatory synapse. C: Negative staining electron micrograph of a late growthphase Bdellovibrio qaytius (arrow) next to a ghost cell of a prey cell (arrow head). The flagellum is detached in preparation for binary fission. D: FISH epifluorescence micrograph of B. qaytius (red with specific FISH probe, arrow) attached to host cell (green DAPI stained, arrow head). E: Thin-section electron micrograph of growth-phase B. qaytius (black arrow) attached to a prey cell (black arrow head). The prey cell has an emptied cytoplasm and shows an invagination of the membrane (white arrow head).F: Thin section micrograph close-up of the predatory synapse shown in E. The membrane of the predator (arrow) and the prey cell (arrow head) remain intact, but show electron dense signatures. G: growth-phase B. qaytius (black arrow) showing polar attachment to a prey cell (black arrow head) that also shows an invaginating cell membrane (white arrow head). Scale bar in D: $2.5 \mu \mathrm{m}$, other scale bars $+500 \mathrm{~nm}$.

\section{Bdellovibrio qaytius has a complex genome for an epibiotic predator}

\section{Genome structure and content}

The 3,348,710-bp B. qaytius genome is similar in size to periplasmic Bdellovibrio spp., but considerably larger than that of Bdellovibrio exovorus, another epibiotic predator with a 2.66 $\mathrm{Mb}$ genome and with $38.9 \%$ also has the lowest GC content of any species within the genus. The GC content is relatively constant and exhibits a dichotomy in GC-skew that is typical of a circular bacterial genome (Figure 3A). Furthermore, the B. qaytius genome contains one 
258 RNAs (ssrS, rnpB and ffs), and three putative riboswitches (Figure 3B). A total of 3166 protein

259 coding genes were identified and are distributed equally between the plus and minus

260 strands(Figure 3A). These proteins represent 22 different functional clusters of orthologous 261 genes (Figure 3B).

A

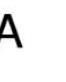

Figure 3: Bdellovibrio qaytius genome. A: Genomic map of B. qaytius. From outside to inwards: Plus-strand CDS (light blue), Minus-strand CDS (dark blue), tRNAs (black), rRNAs (red), and non-coding RNAs (pink), GC-content (purple/mustard), and GC-skew (red/green). B: Abundance of 866 identified functional clusters of orthologous genes in the B. qaytius genome

\section{Metabolism}

The $B$. qaytius genome encodes a metabolism typical for a predatory bacterium. Glycolysis and the complete TCA cycle, as well as a core set of pentose phosphate pathway genes are present, suggesting B. qaytius is capable of several sugar conversions, and is able to provide the precursors for riboflavin biosynthesis. Pyruvate metabolism is coded for, but propanoate metabolism is only partially possible. A vitamin B6 biosynthesis pathway is encoded and acetyl-CoA biosynthesis is possible via pantoate. Nicotinamide metabolism and biosynthesis pathways also exist. Oxidative phosphorylation is encoded with the exception of cytochrome C reductase. The presence of core mevalonate pathway enzymes suggests this pathway is functional.

Based on inferred CDS, B. qaytius can synthesize pyrimidines and purines de novo, and in contrast to other epibiotic predators, produce inosine. A complete DNA polymerase complex 
facilitates DNA replication. As well, all types of DNA repair pathways are present, including

280 base excision, nucleotide excision, mismatch repair and homologous recombination, the latter

281 being limited to single-stand-break repair.

282 Bdellovibrio qaytius encodes a complete ribosome except for the non-essential protein

283 L28, and there is a complete set of tRNAs loaded by aminoacyl-tRNA-synthetases for every

284 amino acid. The genome encodes a core RNA degradasome for post-transcriptional regulation

285 and nucleotide recycling. Amino-acid biosynthesis pathways are limited and only cysteine,

286 methionine, glutamate, lysine, proline and threonine can be completely synthesized de novo.

287 Glycine and serine can be synthesized via the one-carbon pool pathway using tetrahydrofolate.

288 Aspartate, alanine leucine, isoleucine, valine phenylalanine and tyrosine can be converted from

289 their direct precursors, which are presumably acquired from the prey.

290 Complete fatty-acid degradation pathways are coded for, but fatty-acid elongation seems limited.

291 Also encoded are complete sec and gsp pathways for protein secretion, as well as a partial tat

292 pathway (subunits tatA, tatC, tatD). Extensive peptidoglycan production, as well as partial

293 lipopolysaccharide biosynthesis pathways putatively decorate the periplasmic space and cell

294 surface.

\section{Regulatory elements}

Master regulators, such as sigma factor 28 / FliA, which is proposed to enable the switch between attack and growth-phase modes in other Bdellovibrio species, is present in B. qaytius

298 and might work alongside putative riboswitch elements similar to those found in B. bacterivorus

299 (6). No homologue of the host interaction ("hit") locus protein bd0108, of B. bacterivorus, was

300 found in B. qaytius, suggesting that it may deploy a different pilus regulation mechanism, and

301 therefore might not be able to switch between facultative and obligate predation.

\section{Predatory arsenal}

304 biosynthesis pathway for flagellar assembly and regulation provides locomotion in the attack-

305 phase. Attack phase cells are likely guided by a canonical, almost, complete chemotaxis pathway 306 that is only missing cheY. Substrate recognition is putatively mediated by tatC and two copies of 307 von Willebrand factors (24). A type IV pilus appears to be present that is putatively involved in 308 prey-cell attachment through pilZ. To access resources within the prey, an array of transporters 309 are coded for, many of which show signal peptides that facilitate export, and might insert into the 
310 prey-cell membrane. ABC-type transporters likely import phosphate (pst), phosphonate (phn),

311 and lipopolysaccharides (lpt), while there appears to be partial ABC transporter systems for

312 lipoproteins, thiamine, branched-chain amino acids, oligo and dipeptides, microcin,

313 phospholipids, biotin, daunorubiscine, alkylophaosphate, methionine, iron and siderophores,

314 cobalt, sugar and organic solvents. Non-ABC transporter system CDS are present for potassium

315 (kdp), biopolymers (exbD and tol), iron (ofeT), heavy metals (cusA), biotin (bioY), threonine

316 (rhtB), as well as for several multidrug exporters (bcr, cflA, arcB). CDS for low-specificity

317 transporters include MFS and EamE transporters, as well as transporter for ions and cations,

318 macrolide, chromate, as well as sodium-dependent transporters and others of uncharacterized

319 specificity.

\section{Bdellovibrio qaytius is a basal representative of its genus}

In phylogenetic analysis of the $16 \mathrm{~S}$ rDNA locus, B. qaytius occupies a well-supported basal branch within the genus Bdellovibrio; the closest relatives are found in environmental amplicon sequences, as well as in poorly characterized isolates (Figure 4A). Notably, these strains from a tight cluster basal to B. exovorus and B. bacterivorus strains and show rather low boot-strap support within their clade despite being closely related. This cluster appears to be equivalent to the "cluster 2" described by Davidov et al. (7).

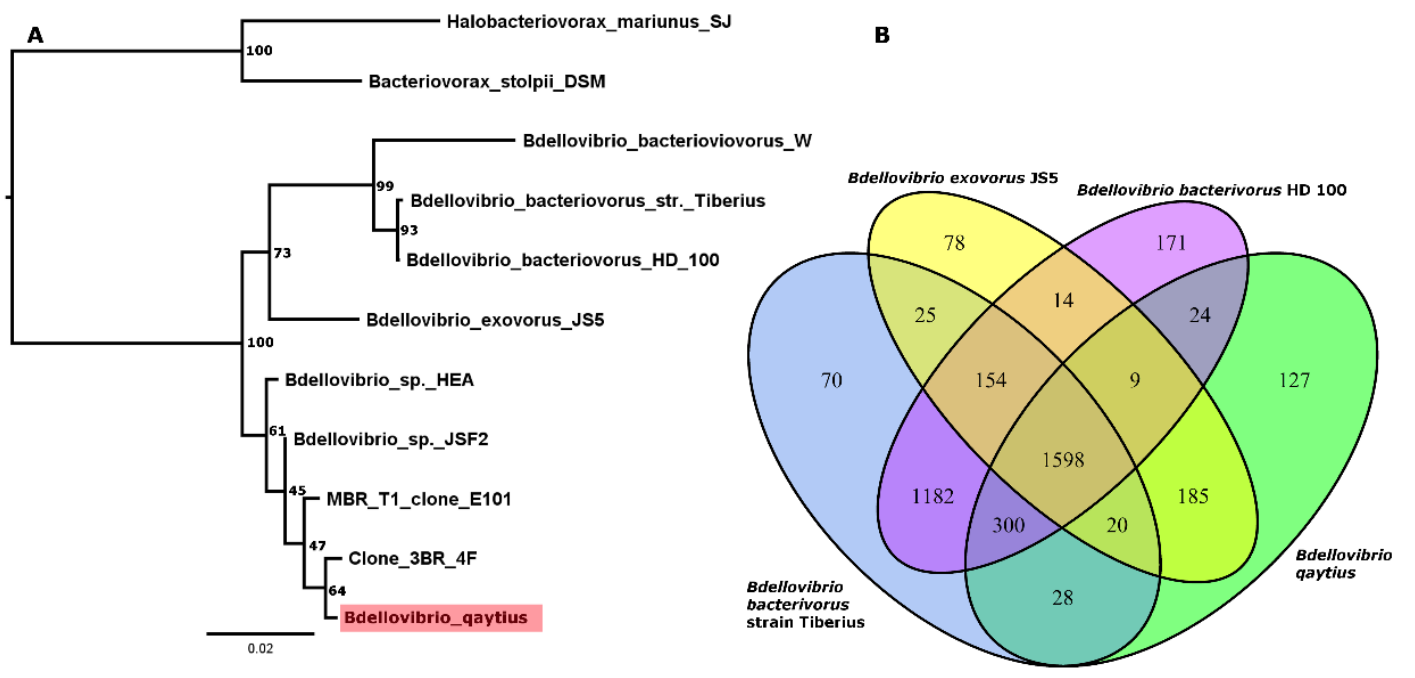

Figure 4 Bdellovibrio qaytius phylogenetic placement. A: $16 \mathrm{~S}$ phylogenetic tree showing the completely sequenced Bdellovibrio species as well as the top BLAST hits to Bdellovibrio qaytius from uncharacterized isolates, as well as from metagenomic data. B: Shared gene cluster analysis 
Shared gene cluster analysis was congruent with the 16S phylogeny with the majority of gene clusters in B. qaytius being shared with other members of the genus Bdellovibrio (Figure

334 4B). Surprisingly, B. qaytius shares more than 300 gene clusters with periplasmic predators, 335 which are not found in the epibiotic predator B. exovorus, despite their similar predation strategy.

336 On the other hand, the epibiotic predators B. qaytius and B. exovorus shared more than 130 genes 337 that are not found in periplasmic predators, and might be involved in epibiotic predation. These 338 exclusively epibiotic genes within members of the genus Bdellovibrio include CDS for proteases, 339 peroxiredoxin, glutathione-dependent formaldehyde-activating enzyme, nucleases, hydrolases 340 thioesterase, polysaccharide deacetylase, an amino-acid ABC transporter, as well as many poorly 341 characterized proteins. Gene clusters exclusively shared with B. exovorus, as well as the 342 phylogenetically distant $M$. aeruginosavorus that also deploys an epibiotic predation strategy, are 343 remarkable as they might highlight a common set of genes are required for epibiotic predation.

344 Six such genes that were identified include anhydro-N-acetylmuramic acid kinase, peptidoglycan 345 translocase, a FAD/NAD binding protein, a cation-transporting P-type ATPase and a 346 pseudouridine synthase.

Discussion

Bdellovibrio qaytius is an epibiotic predator of the beta-proteobacterium Paraburkholderia fungorum

While B. qaytius 16S rDNA was detected by PCR in liquid cultures of Pseudomonas fluorescence as well as E. coli after two rounds of propagation, a weak signal compared to 353 cultures containing Paraburkholderia fungorum as prey suggests the passive carry-over of $B$. 354 qaytius cells or genetic material in the absence of replication (Supplementary Figure 1). This is 355 in line with the higher density of putative attack-phase B. quaytius cells observed in P. fungorum 356 cultures compared to the other putative hosts. Ultimately, plaque assay confirmed the beta357 proteobacterium Paraburkholderia fungorum as the only host organism of B. qaytius observed in 358 the present study. periplasmic predators.

Microscopic analysis clearly shows B. qaytius deploying an epibiotic predation strategy, which is reflected in its genome that shares several features previously identified to be involved 
363 in epibiotic predators such as B. exovorus and Micavibrio aeruginosavorus. These include

364 physical features such as the number of rDNA loci, as well as metabolic capabilities based on

365 gene content that suggests limited fatty-acid elongation and the absence of polyhydroxyalkanoate

366 depolymerase and the siderophore aerobactin, all present in periplasmic predators (11). In

367 contrast, $B$. qaytius also has coding sequences for the biosynthesis of isoleucine and tyrosine, as

368 well as for riboflavin and vitamin B6, which had been found in periplasmic but not epibiotic

369 predators reflecting its comparatively large and complex genome (10). The linkage of these

370 genes with periplasmic replication was by association and not for functional reasons; hence, the

371 presence of several of these genes in B. qaytius may simply reflect its relatively larger genome

372 size. Cluster analysis of orthologous genes in B. qaytius, other Bdellovibrio spp. as well as the

373 unrelated BALOs Micavibrio aeruginosavorus and Halobacteriovorax marinus reveals just six

374 gene clusters associated with epibiotic predation. Strikingly, these genes suggest that the prey

375 peptidoglycan is salvaged by $\mathrm{N}$-acetylmuramic-acid kinase as well as a peptidoglycan

376 translocase that is specific to epibiotic predators. Since this limited complement of genes was

377 found between distantly related taxa, there might not be a clear functional separation between

378 epibiotic and periplasmic predators. This is consistent with epibiotic predation evolving

379 independently within the genera Bdellovibrio and Micavibrio. Therefore, conclusions regarding

380 function based on gene-cluster analysis should be interpreted with caution, especially since

381 functionally equivalent proteins can group into different gene clusters, and thus may escape such

382 analysis. Accordingly, gene-cluster comparison among species in the same genus that deploy

383 different predation strategies is more informative and revealed a specialized complement of

384 proteases nucleases, hydrolases and detoxifying enzymes in epibiotic Bdellovibrio species

385 (Figure 4 B). Notably, the addition of B. qaytius as a second epibiotic Bdellovibrio species

386 greatly decreased the number of genes associated with epibiotic predation, as its large genome

387 has greater overlap with periplasmic Bdellovibio species. Further, this suggests that different

388 mechanisms can be deployed in both, epibiotic and periplasmic predation.

Epibiotic predation is a common strategy of environmental Bdellovibrio species and could be the ancestral phenotype

393 "commercial aquaculture preparations", soils, waste-water activated sludge, and iron-oxidizing 
394 freshwater environments (7, 25-27). This broad diversity of habitats suggests that Bdellovibrio

395 species that are closely related to B. qaytius are widely distributed in freshwaters, and could be a

396 major contributor to global BALO diversity. Because of the phylogenetic placement and the

397 broad distribution of related isolates, epibiotic predation might be common among BALOs,

398 despite being underrepresented in isolates. The recurrent detection of populations of B. qaytius in

399 the pond from which it was isolated confirms that is part if the natural community and therefore

400 supports the idea that epibiotic predation might be common in nature. Additionally, both $B$.

401 qaytius and B. exovorus are epibiotic and branch basal within the genus, compared to the

402 periplasmic predators. This suggests that epibiotic predation might be the ancestral predation

403 type in the genus.

404 Detailed knowledge of environmental BALO diversity is still missing. The discovery and 405 analysis of Bdellovibrio qaytius provides new insights into this fascinating group. Its 406 intermediate genomic complement blurs the lines between what is required for epibiotic and 407 periplasmic replication. Moreover, as a representative of a basal and widespread member within 408 the genus, Bdellovibrio, it suggests that epibiotic predation is common in the environment, and 409 might be the ancestral form of predation in this genus.

\section{Acknowledgements:}

412 The work was supported by grants to C. S. from the Natural Sciences and Engineering 413 Research Council of Canada (NSERC; 05896), Canada Foundation for Innovation (25412), 414 British Columbia Knowledge Development Fund, and the Canadian Institute for Advanced 415 Research (IMB). C. D. was supported in part by a fellowship from the German Academic 416 Exchange Service (DAAD). T. L. was supported in part by an award by the Natural Sciences and

417 Engineering Research Council of Canada. The authors would like to thank Jill Campbell, the 418 coordinator for the Musqueam Language and Culture Department 419 (https://fnel.arts.ubc.ca/community/musqueam-nation/musqueam-language-and-culture/), for her 420 guidance in identifying a suitable species name based on the hən $\square \mathrm{q} \square$ əmin $\square$ əm $\square$ language. 


\section{Literature}

424 1. Weinbauer MG, Höfle MG. 1998. Significance of viral lysis and flagellate grazing as 
Bdellovibrio by fluorescence in situ hybridization. Applied and environmental microbiology 73:7488-7493.

17. Delcher AL, Harmon D, Kasif S, White O, Salzberg SL. 1999. Improved microbial gene identification with GLIMMER. Nucleic acids research 27:4636-4641.

18. Käll L, Krogh A, Sonnhammer EL. 2004. A combined transmembrane topology and signal peptide prediction method. Journal of molecular biology 338:1027-1036.

19. Moriya Y, Itoh M, Okuda S, Yoshizawa AC, Kanehisa M. 2007. KAAS: an automatic genome annotation and pathway reconstruction server. Nucleic acids research 35:W182W185.

20. Karp PD, Paley SM, Krummenacker M, Latendresse M, Dale JM, Lee TJ, Kaipa P, Gilham F, Spaulding A, Popescu L. 2009. Pathway Tools version 13.0: integrated software for pathway/genome informatics and systems biology. Briefings in bioinformatics 11:40-79.

21. Edgar RC. 2004. MUSCLE: multiple sequence alignment with high accuracy and high throughput. Nucleic acids research 32:1792-1797.

22. Stamatakis A. 2014. RAxML version 8: a tool for phylogenetic analysis and postanalysis of large phylogenies. Bioinformatics 30:1312-1313.

23. Li L, Stoeckert CJ, Roos DS. 2003. OrthoMCL: identification of ortholog groups for eukaryotic genomes. Genome research 13:2178-2189.

24. Alami M, Lüke I, Deitermann S, Eisner G, Koch H-G, Brunner J, Müller M. 2003. Differential interactions between a twin-arginine signal peptide and its translocase in Escherichia coli. Molecular cell 12:937-946.

25. Wen C, Xue M, Zhang J, Huang Y, Zhou S. 2009. The detection of Bdellovibrio-andlike organisms in commercial preparations used for aquaculture. Journal of Fisheries of China 33:326-333.

26. D'Anteo S, Mannucci A, Meliani M, Verni F, Petroni G, Munz G, Lubello C, Mori G, Vannini C. 2015. Nitrifying biomass characterization and monitoring during bioaugmentation in a membrane bioreactor. Environmental technology 36:3159-3166.

27. Duckworth OW, Holmström SJ, Peña J, Sposito G. 2009. Biogeochemistry of iron oxidation in a circumneutral freshwater habitat. Chemical Geology 260:149-158. 


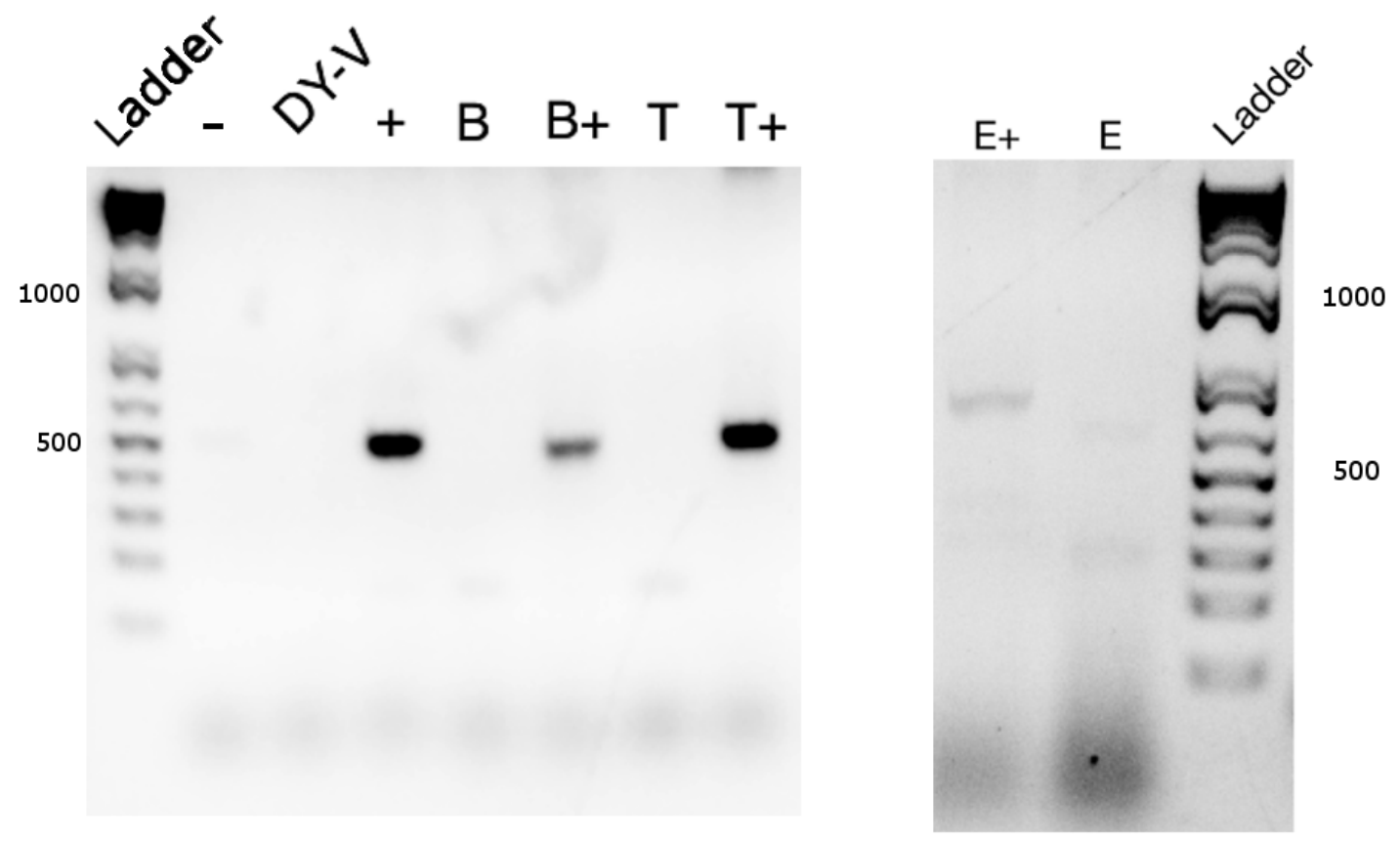

502 Supplementary Figure 1: Bdellovibrio qaytius host range.B. qaytius propagation was assessed by 503 PCR using strain-specific primers: -= water control, DY-V=medium control, += Positive control from mixed Nitobe garden pond assemblage culture, $\mathrm{B}=$ Pseudomonas fluorescence culture, $\mathrm{B}+=$ Pseudomonas fluorescence culture after inoculation and two propagation cycles showing carry over effects of $B$. qaytius, $\mathrm{T}=$ Paraburkholderia fungorum culture, $\mathrm{T}+=$ Paraburkholderia fungorum culture after inoculation and two times propagation of B. qaytius showing active replication. $\mathrm{E}+=E$. coli culture after inoculation and two propagation cycles showing carry over 

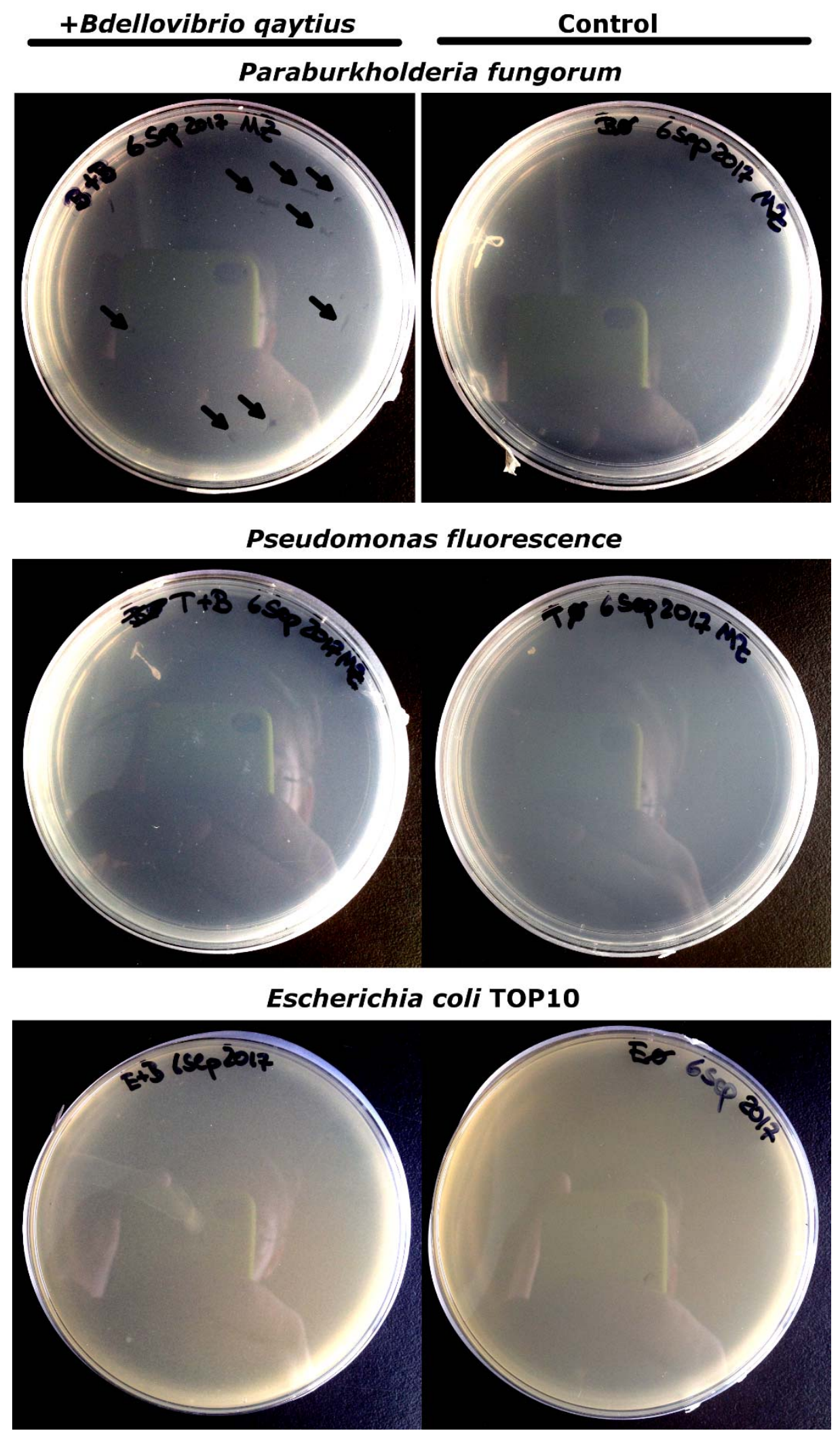

512 Supplementary Figure 2: Bdellovibrio qaytius plaque assay. Left column shows B. qaytius 513 infected plates, right column shows the control. Plaques highlighted by arrows. 


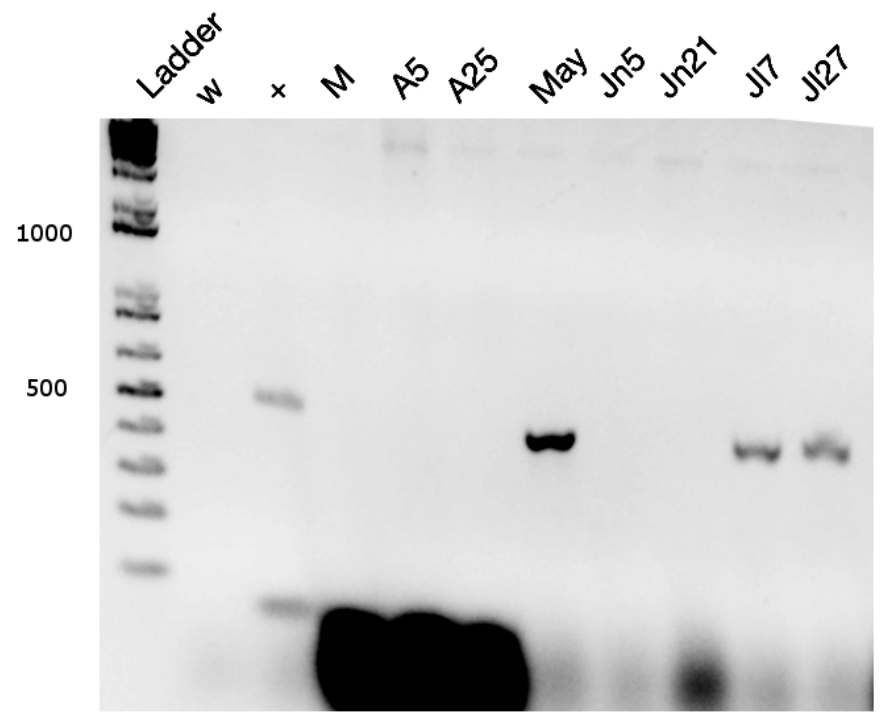

515 Supplementary Figure 3: Bdellovibrio qaytius continued presence in Nitobe Gardens pond.:

516 Bdellovibrio qaytius detection at various time points with B. qaytius specific primers in samples 517 collected from Nitobe gardens UBC: $\mathrm{w}=$ water control, $+=$ positive control, $\mathrm{M}=23$ March 2017 , 518 A5= 5 April 2017, A25= 25 April 2017, May= 15 May 2017,Jn5= 5 June 2017, Jn21= 21 June $5192017, \mathrm{~J} 17=7$ July 2017, J127= 27 July 2017. 